\title{
CATALISADORES DE FE PARA A CONVERSÃO DE METANO A FORMALDEÍDO
}

\author{
M. E. A. FERNANDES ${ }^{1}$, L. TRAVALLONI ${ }^{1}$, M. A. P. DA SILVA ${ }^{1}$ \\ ${ }^{1}$ Universidade Federal do Rio de Janeiro, Departamento de Engenharia de Química \\ E-mail para contato: monica@eq.ufrj.br
}

\begin{abstract}
RESUMO - O metano é o principal componente do gás natural, uma matéria-prima abundante. Sua conversão em metanol é realizada via produção de gás de síntese, um processo energeticamente intensivo. Assim, a síntese de compostos oxigenados (metanol e formaldeído) pela oxidação parcial de metano (OPM) é uma alternativa promissora. Dentre os catalisadores de OPM mais estudados, estão os de ferro suportado em zeólitas. Neste trabalho, avaliou-se o desempenho na OPM de catalisadores Fe/ZSM-5, modificados ou não pela adição de fósforo. Diversos teores de Fe e $\mathrm{P}$ foram impregnados na ZSM-5 a partir de soluções de $\mathrm{Fe}\left(\mathrm{NO}_{3}\right)_{3}, \mathrm{H}_{3} \mathrm{PO}_{4}$ ou $\left(\mathrm{NH}_{4}\right)_{2} \mathrm{HPO}_{4}$. Os catalisadores obtidos foram caracterizados por TPR e DRS. Testes catalíticos foram realizados a $500{ }^{\circ} \mathrm{C}$ usando-se $\mathrm{N}_{2} \mathrm{O}$ como oxidante (razão $\mathrm{CH}_{4} / \mathrm{N}_{2} \mathrm{O}=2$ ). A adição de $1 \%$ de $\mathrm{P}$ aumentou a produtividade inicial de formaldeído, mas diminuiu a estabilidade dos catalisadores. Dentre os precursores de $\mathrm{P}, \mathrm{o}\left(\mathrm{NH}_{4}\right)_{2} \mathrm{HPO}_{4}$ proporcionou uma maior produtividade de formaldeído.
\end{abstract}

\section{INTRODUÇÃO}

Um dos maiores desafios da catálise é a oxidação parcial de metano (OPM) visando à síntese de compostos oxigenados, como metanol e formaldeído. O metano é o principal componente do gás natural, uma matéria-prima abundante. Atualmente, a conversão de metano a metanol é realizada em duas etapas, por intermédio da produção de gás de síntese, um processo intensamente endotérmico. Assim, muitos pesquisadores estão buscando meios de converter o metano a oxigenados em uma única etapa exotérmica. Os resultados de diversas pesquisas até o presente momento foram insatisfatórios, pois elevadas seletividades só foram obtidas para baixas conversões de metano, o que inviabiliza aplicações industriais. Isso se deve à oxidação consecutiva dos oxigenados a $\mathrm{CO}$ e $\mathrm{CO}_{2}$, termodinamicamente mais estáveis. $\mathrm{O}$ desafio na OPM consiste em impedir o progresso das reações em série desencadeadas nesse processo (Fajardo et al., 2008).

A OPM é geralmente feita com $\mathrm{O}_{2}$ ou $\mathrm{N}_{2} \mathrm{O}$, a $400-750{ }^{\circ} \mathrm{C}$, faixa em que são apresentadas atividades catalíticas mais expressivas. À pressão atmosférica, catalisadores desempenham um papel importante na OPM. Os catalisadores mais estudados são óxidos de ferro suportados em sílica e zeólitas (Barbero et al., 2001; Fajardo et al., 2008). A acidez do suporte pode aumentar a conversão consecutiva do formaldeído formado e, portanto, reduzir a seletividade a este oxigenado (Wang et al., 2004). Assim, catalisadores modificados por aditivos que reduzem a acidez (como fósforo) podem 


\section{9 a 22 de outubro de 2014 \\ Florianópolis/SC}

apresentar maior seletividade a oxigenados.

O objetivo deste trabalho foi estudar a OPM empregando catalisadores de Fe suportado em ZSM-5. Foi avaliado o efeito da adição de diferentes teores de $\mathrm{P}$ aos catalisadores sobre seus desempenhos na reação. Os efeitos do tipo de precursor de $\mathrm{P}$ e do método de incorporação do $\mathrm{Fe}$ (impregnação ou troca iônica) também foram avaliados.

\section{EXPERIMENTAL}

\subsection{Síntese de catalisadores}

Impregnação úmida: Dois lotes de ZSM-5 foram utilizados, denominados HZA e HZB. Foram sintetizados cinco catalisadores de Fe/ZSM-5, com ou sem P: $0,5 \%(\mathrm{~m} / \mathrm{m})$ de $\mathrm{Fe}(0.5 \mathrm{FeHZA})$, $2 \%(\mathrm{~m} / \mathrm{m})$ de Fe $(2 \mathrm{FeHZA}), 0,5 \%(\mathrm{~m} / \mathrm{m})$ de Fe e $4 \%(\mathrm{~m} / \mathrm{m})$ de P $(4 \mathrm{P} 0.5 \mathrm{FeHZA}), 0,5 \%(\mathrm{~m} / \mathrm{m})$ de Fe e $1 \%(\mathrm{~m} / \mathrm{m})$ de P $(1 \mathrm{P} 0.5 \mathrm{FeHZA})$ e $2 \%(\mathrm{~m} / \mathrm{m})$ de Fe e $1 \%(\mathrm{~m} / \mathrm{m})$ de P $(1 \mathrm{P} 2 \mathrm{FeHZB})$. A impregnação de P na ZSM-5 foi feita antes da impregnação de Fe, a partir de uma solução aquosa de $\mathrm{H}_{3} \mathrm{PO}_{4}$. Após a adição dessa solução à zeólita, a suspensão resultante foi mantida sob agitação à temperatura ambiente por $12 \mathrm{~h}$ e, em seguida, foi seca a $70{ }^{\circ} \mathrm{C}$ sob vácuo parcial. $\mathrm{O}$ sólido obtido foi mantido por $24 \mathrm{~h}$ em estufa a $100{ }^{\circ} \mathrm{C}$ e calcinado em mufla a $550{ }^{\circ} \mathrm{C}$ por $1 \mathrm{~h}$. A impregnação de $\mathrm{Fe}$ no material calcinado seguiu a mesma metodologia, usando-se uma solução aquosa de $\mathrm{Fe}\left(\mathrm{NO}_{3}\right)_{3}$. Além disso, foram sintetizados dois catalisadores suportados em ZSM-5 proveniente de outro lote, denominado HZC, ambos contendo $2 \%(\mathrm{~m} / \mathrm{m})$ de Fe e $1 \%(\mathrm{~m} / \mathrm{m})$ de P. Num desses catalisadores, o precursor de P foi $\mathrm{o} \mathrm{H}_{3} \mathrm{PO}_{4}$ (1P2FeHZCA); no outro catalisador, foi o sal $\left(\mathrm{NH}_{4}\right)_{2} \mathrm{HPO}_{4}$ (1P2FeHZCS). O procedimento das impregnações sucessivas foi idêntico ao descrito anteriormente.

Troca iônica via radiação de micro-ondas: Foi preparado um catalisador com $0,5 \%(\mathrm{~m} / \mathrm{m})$ de $\mathrm{Fe}$ suportado em ZSM-5 (HZB), denominado 0.5FeHZB, usando-se um equipamento CEM, modelo Discover System, e uma solução aquosa de $\mathrm{Fe}\left(\mathrm{NO}_{3}\right)_{3}$. A troca iônica foi realizada em uma única etapa a $150{ }^{\circ} \mathrm{C}$, por $1 \mathrm{~h}$. A suspensão resultante foi filtrada a vácuo. $\mathrm{O}$ sólido foi seco em estufa a $100{ }^{\circ} \mathrm{C}$ por $24 \mathrm{~h}$ e calcinado em mufla a $550{ }^{\circ} \mathrm{C}$ por $1 \mathrm{~h}$.

\subsection{Caracterização de catalisadores}

Análises de fluorescência de raios-X (FRX) foram feitas em espectrômetro Rigaku, modelo Rix 3100, com tubo de ródio (4 kW). Análises de difração de raios-X (DRX) foram feitas em difratômetro Rigaku Miniflex (30 kV e $15 \mathrm{~mA})$ com tubo de $\mathrm{Cu}(1,5417 \AA)$, em intervalos de $2 \theta=0,05^{\circ}$ e com $2 \mathrm{~s}$ por passo. Análises texturais foram feitas a partir de medidas da adsorção física de $\mathrm{N}_{2} \mathrm{a}-196{ }^{\circ} \mathrm{C} \mathrm{em}$ equipamento TriStar 3000 da Micromeritics; as amostras foram pré-tratadas a $300{ }^{\circ} \mathrm{C}$ sob vácuo, por $18 \mathrm{~h}$. Ensaios de redução a temperatura programada (TPR) foram feitos num equipamento com detector de condutividade térmica; as amostras foram pré-tratadas a $500{ }^{\circ} \mathrm{C}\left(10{ }^{\circ} \mathrm{C} / \mathrm{min}\right)$ por $30 \mathrm{~min}$, sob $30 \mathrm{~mL} / \mathrm{min}$ de argônio, e a redução foi feita com uma mistura de $10 \% \mathrm{H}_{2} / \mathrm{Ar}(30 \mathrm{~mL} / \mathrm{min})$ e aquecimento até $800^{\circ} \mathrm{C}\left(10^{\circ} \mathrm{C} / \mathrm{min}\right)$. Análises de espectroscopia de refletância difusa (DRS) na região do UV-Vis foram realizadas em espectrofotômetro Cary 5000, utilizando-se como referência a ZSM-5 sem $\mathrm{Fe}$, contendo o mesmo teor de $\mathrm{P}$ do catalisador analisado. 


\subsection{Testes catalíticos}

A OPM foi realizada em reator tubular de quartzo com leito fixo de catalisador, à pressão atmosférica e a $500{ }^{\circ} \mathrm{C}$. A alimentação do reator continha uma razão molar $\mathrm{CH}_{4} / \mathrm{N}_{2} \mathrm{O}=2$ e a velocidade espacial foi de $5,9 \mathrm{~mol} \mathrm{~h}^{-1} \mathrm{~g}^{-1}$. O catalisador foi pré-tratado a $500{ }^{\circ} \mathrm{C}\left(10{ }^{\circ} \mathrm{C} / \mathrm{min}\right)$ sob 30 $\mathrm{mL} / \mathrm{min}$ de He. O efluente reacional foi analisado em cromatógrafo Agilent $6890 \mathrm{~N}$ equipado com duas colunas em série (Plot Q e peneira molecular) e detector de condutividade térmica.

\section{RESULTADOS E DISCUSSÃO}

\subsection{Caracterização de catalisadores}

A Tabela 1 apresenta os teores de $\mathrm{P}$ e Fe, a cristalinidade relativa (CR), a área específica (A) e o volume de microporos específico (VP) de cada catalisador. A razão molar $\mathrm{SiO}_{2} / \mathrm{Al}_{2} \mathrm{O}_{3}$ (SAR) é similar para todos os suportes (23-27). Além disso, as composições da HZA e da HZB são muito similares, mas a zeólita HZC apresentou um teor maior de $\mathrm{Na}(0,4 \% \mathrm{~m} / \mathrm{m}$, uma ordem de grandeza maior em relação aos outros lotes de ZSM-5), o que deve estar relacionado a uma menor acidez.

Tabela 1 - Composição química, cristalinidade relativa e propriedades texturais dos catalisadores.

\begin{tabular}{|c|c|c|c|c|c|}
\hline Catalisador & $\mathrm{P}(\% \mathrm{~m} / \mathrm{m})$ & $\mathrm{Fe}(\% \mathrm{~m} / \mathrm{m})$ & $\mathrm{CR}(\%)$ & $\mathrm{A}^{1}\left(\mathrm{~m}^{2} / \mathrm{g}\right)$ & $\mathrm{VP}^{2}\left(\mathrm{~cm}^{3} / \mathrm{g}\right)$ \\
\hline HZA & - & - & 100 & 370 & 0,13 \\
0.5FeHZA & - & 0,7 & 86 & 382 & 0,20 \\
2FeHZA & - & 2,5 & 79 & 326 & 0,18 \\
1P0.5FeHZA & 1,4 & 0,6 & 106 & 349 & 0,11 \\
4P0.5FeHZA & 5,1 & 0,6 & 70 & 196 & 0,08 \\
\hline HZB & - & - & 100 & - & - \\
0.5FeHZB & - & 0,6 & 91 & 347 & 0,11 \\
1P2FeHZB & 1,3 & 2,0 & 82 & 223 & 0,08 \\
\hline HZC & - & - & 100 & - & - \\
1P2FeHZCA & 1,3 & 2,3 & 83 & 322 & 0,11 \\
1P2FeHZCS & 1,3 & 2,3 & 84 & 318 & 0,11 \\
\hline
\end{tabular}

${ }^{1}$ Método BET; ${ }^{2}$ método t-plot.

A cristalinidade relativa foi calculada a partir dos difratogramas das amostras, sendo a razão entre a área do triplete em $2 \theta=23-25^{\circ}$ para o catalisador impregnado e a área para o suporte correspondente (este triplete apresenta a maior intensidade dos picos e a menor influência da água intracristalina). $\mathrm{O}$ aumento dos teores de Fe e $\mathrm{P}$, em geral, resultou na diminuição da cristalinidade, bem como da área específica e do volume de poros. Os resultados sugerem que, para elevados teores de Fe e P, sua dispersão diminui e formam-se depósitos maiores que bloqueiam parcialmente os poros da ZSM-5. Os catalisadores 1P2FeHZCS e 1P2FeHZCA, com os mesmos teores de P e Fe, porém baseados em precursores de $\mathrm{P}$ diferentes, apresentaram propriedades muito similares. 
A Figura 1 apresenta perfis de TPR de catalisadores e na Tabela 2 são apresentados os graus de redução até $500^{\circ} \mathrm{C}$ (temperatura empregada na $\mathrm{OPM}$ ) e até $800^{\circ} \mathrm{C}$.
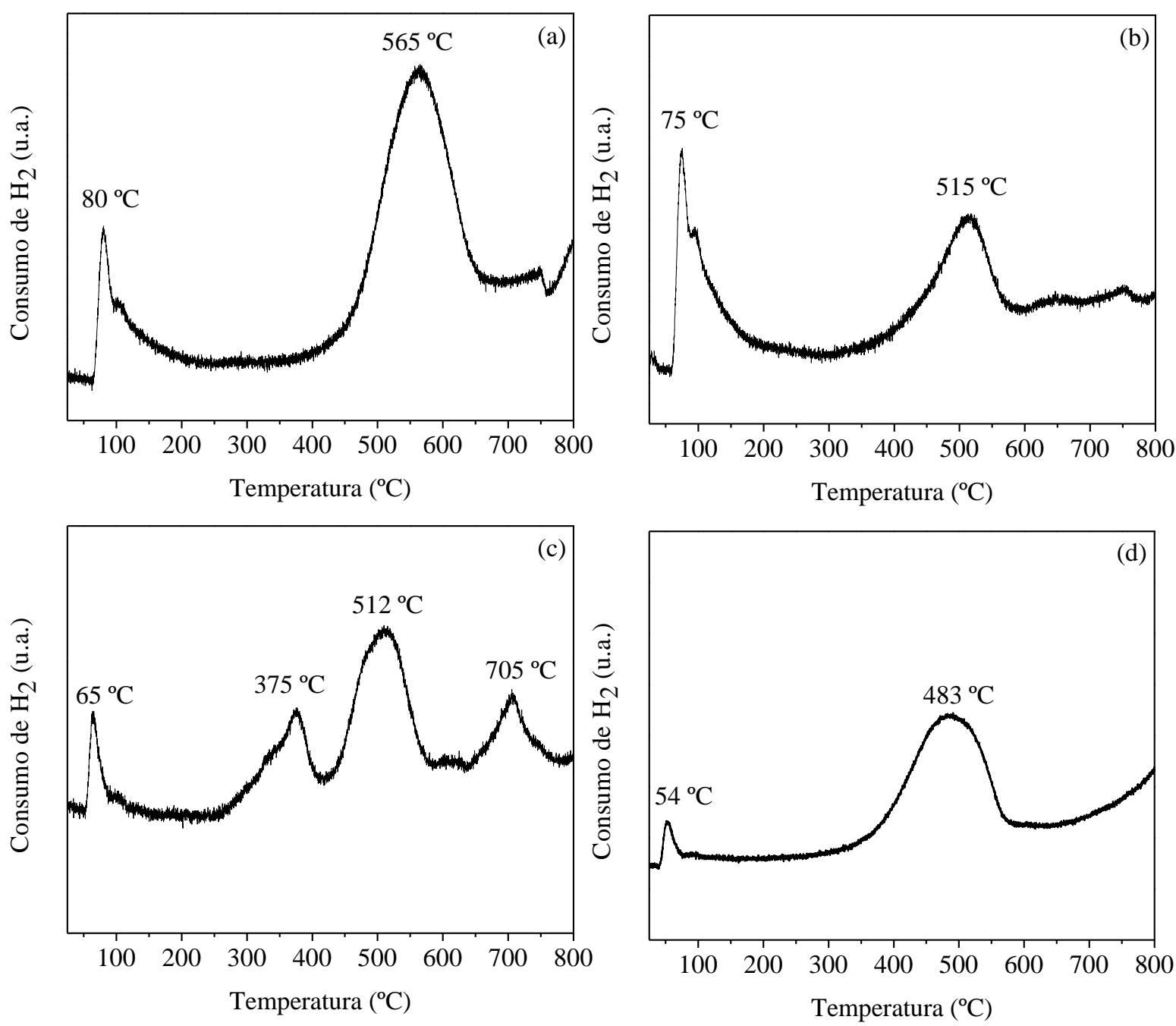

Figura 1 - Perfis de TPR de catalisadores: (a) 4P0.5FeHZA, (b) 1P0.5FeHZA, (c) 2FeHZA e (d) 1P2FeHZB.

Há quatro picos de consumo de $\mathrm{H}_{2}$ para o catalisador sem $\mathrm{P}$ (2FeHZA), atribuídos à redução sequencial de $\mathrm{Fe}^{3+}$ a Fe ${ }^{2+}$ e deste a $\mathrm{Fe}^{0}$ (Li et al., 2010). Para os catalisadores contendo $\mathrm{P}$, há apenas dois picos, indicando que o $\mathrm{P}$ modifica os sítios de Fe. Para o catalisador 4P0.5FeHZA, ambos os picos ocorreram a temperaturas maiores em relação ao 1P0.5FeHZA, sendo o segundo pico mais significativo. Já para o catalisador $1 \mathrm{P} 0.5 \mathrm{FeHZA}$, o primeiro pico foi relativamente maior. Isso indica que o aumento no teor de $\mathrm{P}$ aumentou a concentração de espécies de Fe com menor redutibilidade, o que pode contribuir para a redução da atividade do catalisador no mecanismo redox da OPM. Por outro lado, o grau de redução até $500{ }^{\circ} \mathrm{C}$ foi muito similar para ambos os catalisadores, bem como para o $2 \mathrm{FeHZA}$, enquanto o catalisador $1 \mathrm{P} 2 \mathrm{FeHZB}$ apresentou uma redutibilidade maior. 
Tabela 2 - Graus de redução de catalisadores.

\begin{tabular}{|c|c|c|}
\hline Catalisador & Grau até $500{ }^{\circ} \mathrm{C}(\%)$ & Grau até $800{ }^{\circ} \mathrm{C}(\%)$ \\
\hline 1P0.5FeHZA & 5 & 10 \\
4P0.5FeHZA & 3 & 22 \\
2FeHZA & 6 & 11 \\
\hline 1P2FeHZB & 14 & 20 \\
\hline
\end{tabular}

A Figura 2 apresenta os espectros de DRS dos catalisadores. Os catalisadores 0.5FeHZA, 2FeHZA e 4P0.5FeHZA exibiram uma banda em $270 \mathrm{~nm}$ (o último exibiu também um ombro em 225 $\mathrm{nm})$. O catalisador 0.5FeHZB exibiu uma banda em $225 \mathrm{~nm}$. Os catalisadores 1P0.5FeHZA e 1P2FeHZB exibiram um ombro em 250 e $240 \mathrm{~nm}$, respectivamente. Bandas na faixa 200-250 nm e em 270 nm são relativas a espécies de $\mathrm{Fe}^{3+}$ em coordenação tetraédrica (Berrier et al., 2007).
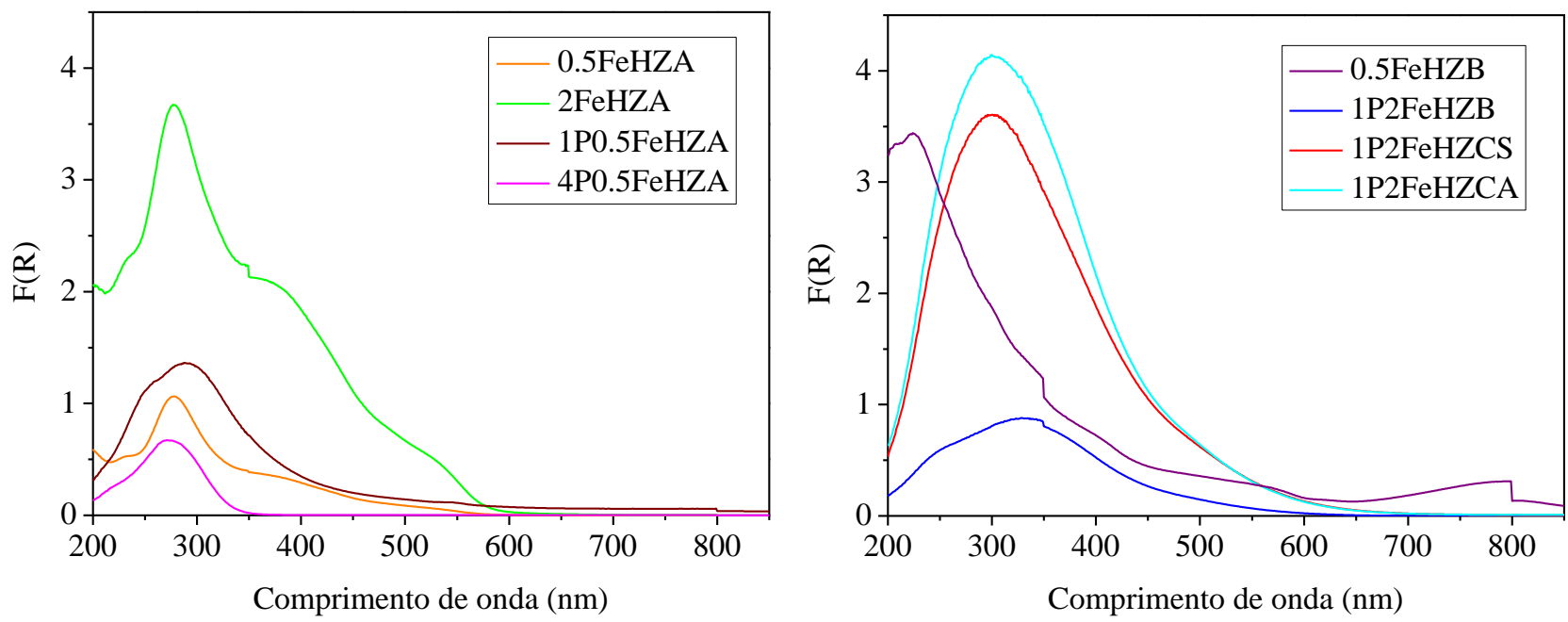

Figura 2 - Espectros de DRS dos catalisadores.

Para o catalisador 1P0.5FeHZA, observa-se um pico em $290 \mathrm{~nm}$, que está relacionado a espécies de $\mathrm{Fe}^{3+}$ em coordenação maior que a tetraédrica (Berrier et al., 2007). O pico em $300 \mathrm{~nm}$ observado para os catalisadores 1P2FeHZCS e 1P2FeHZCA, o pico em $345 \mathrm{~nm}$ para o catalisador 1P2FeHZB e o ombro em $390 \mathrm{~nm}$ para o catalisador 2FeHZA, podem estar relacionados a partículas de $\mathrm{Fe}_{2} \mathrm{O}_{3}$ (Fierro et al., 2011). Bandas em 300-400 nm estão relacionadas a pequenos agregados de $\mathrm{Fe}_{\mathrm{x}} \mathrm{O}_{\mathrm{y}}$ onde íons $\mathrm{Fe}^{3+}$ são coordenados octaedricamente (Kumar et al., 2004); esses agregados se formam para teores relativamente elevados de Fe na zeólita.

\subsection{Testes Catalíticos}

A OPM resultou na formação de $\mathrm{CO}_{\mathrm{x}}$, principalmente, e de pequenas quantidades de formaldeído, em algumas das condições avaliadas. A Figura 3 apresenta a conversão de metano e a produtividade de formaldeído para os catalisadores sintetizados por impregnação. Para o catalisador 4P0.5FeHZA, a conversão foi baixíssima e a formação de formaldeído foi desprezível, o que deve 


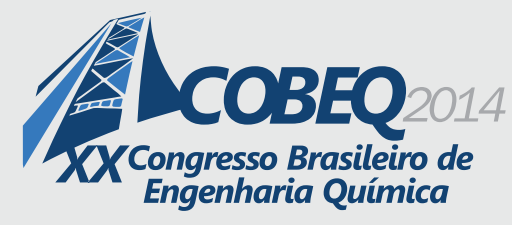

19 a 22 de outubro de 2014
Florianópolis/SC

estar relacionado ao bloqueio de sítios ativos indicado pela análise textural. Já o catalisador 1P0.5FeHZA apresentou conversão inicial semelhante à do catalisador sem $\mathrm{P}$ (0.5FeHZA), porém sofreu desativação nos primeiros $40 \mathrm{~min}$, o que mostra sua instabilidade. Para o catalisador 1P2FeHZB, observou-se uma conversão muito similar à do 1P0.5FeHZA ao longo de toda a reação. Os dois catalisadores com $2 \%(\mathrm{~m} / \mathrm{m})$ de $\mathrm{Fe}$ e $1 \%(\mathrm{~m} / \mathrm{m})$ de $\mathrm{P}$ baseados no mesmo suporte (HZC) apresentaram conversões muito semelhantes até cerca de $1 \mathrm{~h}$ de reação; em seguida, o catalisador 1P2FeHZCA passou a apresentar uma conversão maior que o 1P2FeHZCS, porém ambos exibiram perfis similares. Essa pequena diferença pode estar relacionada a uma menor acidez do catalisador 1P2FeHZCA, à base de $\mathrm{H}_{3} \mathrm{PO}_{4}$ (Blasco et al., 2006). Entretanto, as maiores conversões de metano foram obtidas com os catalisadores não modificados por fósforo.
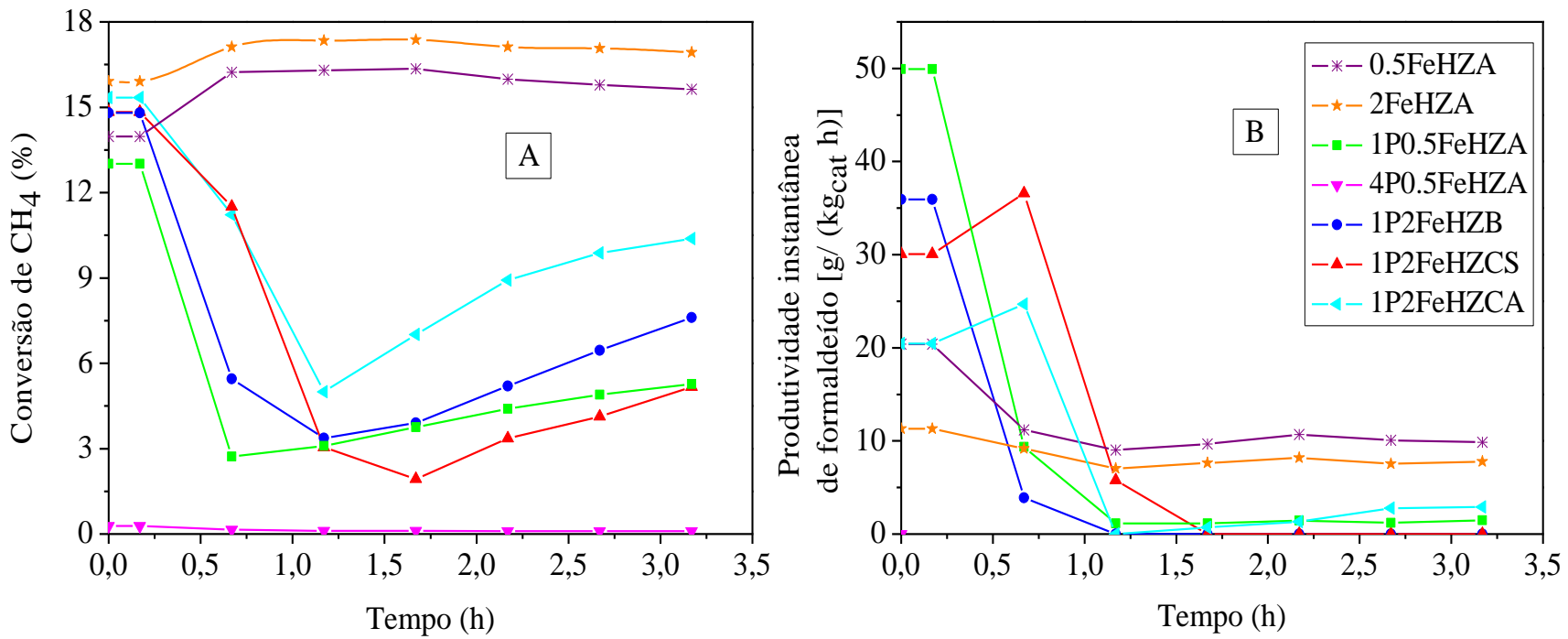

Figura 3 - Conversão de metano (A) e produtividade instantânea de formaldeído (B).

A Tabela 3 apresenta a produtividade acumulada de formaldeído, obtida a partir da integração dos perfis da Figura 3B. O catalisador 0.5FeHZA apresentou a maior produtividade acumulada e mostrou-se estável a partir de 40 min de reação. No início da reação, o catalisador 1P0.5FeHZA apresentou a maior produtividade, mas ela diminuiu rapidamente, indicando que a adição de $1 \%(\mathrm{~m} / \mathrm{m})$ de P diminuiu a estabilidade do catalisador. Comparando os dois catalisadores com $2 \%(\mathrm{~m} / \mathrm{m})$ de Fe e $1 \%(\mathrm{~m} / \mathrm{m})$ de $\mathrm{P}$ baseados no mesmo suporte $(\mathrm{HZC})$, aquele baseado no sal $\left(\mathrm{NH}_{4}\right)_{2} \mathrm{HPO}_{4}$ apresentou melhores resultados que o preparado com $\mathrm{H}_{3} \mathrm{PO}_{4}$, mostrando que a natureza do precursor altera a natureza dos sítios ativos. O catalisador com o maior teor de $\mathrm{P}$ (4P0.5FeHZA) não apresentou formação detectável de formaldeído.

A Figura 4 ilustra a comparação de catalisadores com o mesmo teor de Fe, porém sintetizados por métodos diferentes, em relação à conversão de metano e à produtividade de formaldeído. $\mathrm{O}$ catalisador preparado por impregnação úmida (0.5FeHZA) apresentou maior conversão, que se manteve em torno de $16 \%$ ao longo da reação. Já o catalisador preparado por troca iônica $(0.5 \mathrm{FeHZB})$ apresentou uma conversão inicial inferior e sofreu intensa desativação logo no início da reação. No entanto, a partir de 40 min de reação, a conversão passou a aumentar, indicando uma modificação na 
natureza dos sítios ativos do catalisador $0.5 \mathrm{FeHZB}$.

Tabela 3 - Produtividade acumulada de formaldeído.

\begin{tabular}{|c|c|}
\hline Catalisador & Produtividade $\left(\mathrm{g} / \mathrm{kg}_{\text {cat }}\right)$ \\
\hline 0.5FeHZA & 36 \\
2FeHZA & 26 \\
1P0.5FeHZA & 28 \\
4P0.5FeHZA & 0 \\
\hline 1P2FeHZB & 17 \\
\hline 1P2FeHZCS & 34 \\
1P2FeHZCA & 24 \\
\hline
\end{tabular}
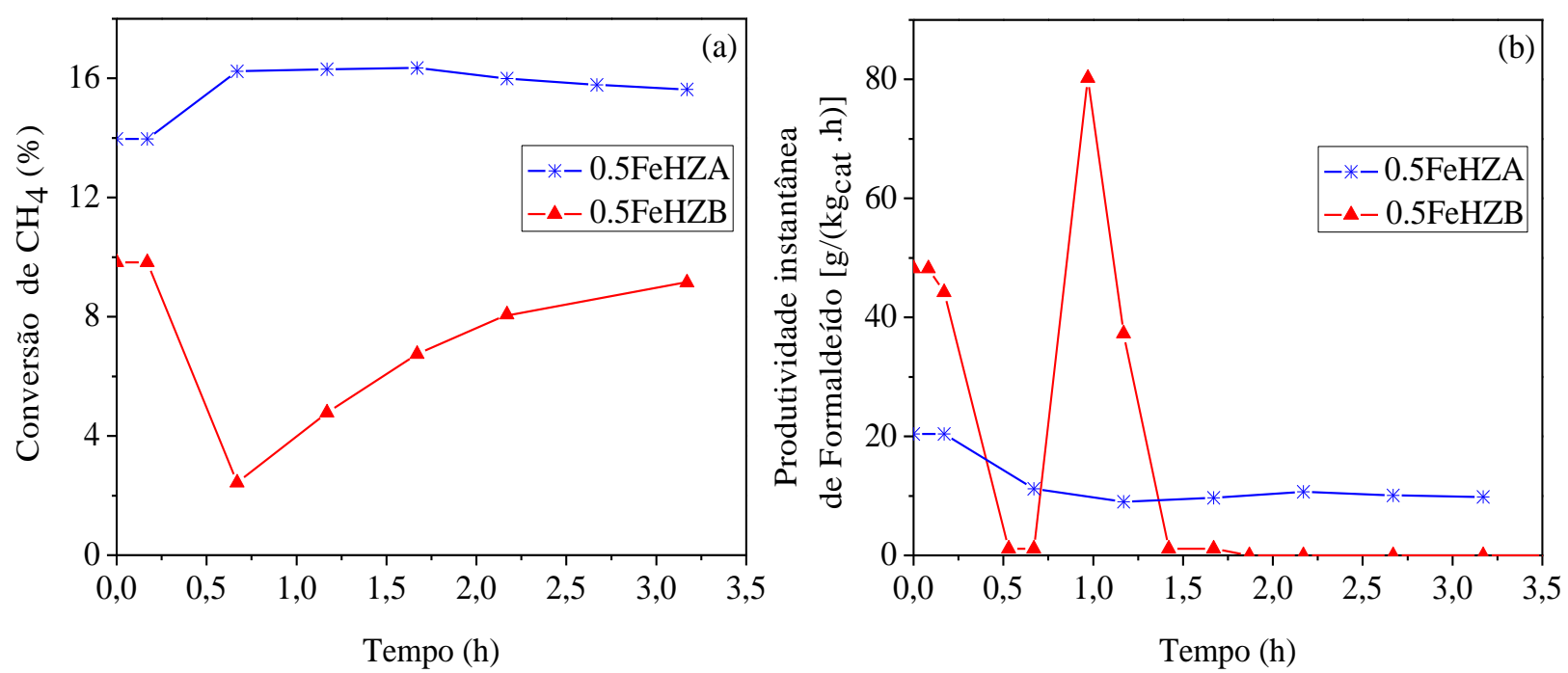

Figura 4 - Conversão de metano (a) e produtividade instantânea de formaldeído (b) para catalisadores preparados por métodos distintos.

O catalisador $0.5 \mathrm{FeHZA}$ apresentou uma produtividade instantânea de formaldeído estável a partir de 40 min de reação. O catalisador 0.5FeHZB apresentou uma produtividade inicial de formaldeído superior à do $0.5 \mathrm{FeHZA}$, porém sofreu uma desativação acentuada nos primeiros 40 min, acompanhando a diminuição na conversão de metano. Nos minutos seguintes, a quantidade formada de formaldeído deixou de ser detectável. Porém, em torno de $1 \mathrm{~h}$ de reação, foi detectada uma formação significativa de formaldeído, que se manteve por poucos minutos. Isso mostra a instabilidade do catalisador preparado por troca iônica via radiação de micro-ondas.

\section{CONCLUSÕES}

Os ensaios de OPM resultaram em baixas conversões de metano e produtividades de 


\section{9 a 22 de outubro de 2014 \\ Florianópolis/SC}

formaldeído nas condições empregadas. O catalisador com $4 \%(\mathrm{~m} / \mathrm{m})$ de P não apresentou atividade. A adição de $1 \%(\mathrm{~m} / \mathrm{m})$ de $\mathrm{P}$ diminuiu a estabilidade dos catalisadores, o que deve estar relacionado à redução da acidez do suporte (HZSM-5), indicando que essa propriedade tem um papel importante no mecanismo da OPM. Dentre os catalisadores impregnados com P a partir de diferentes precursores, aquele baseado em $\left(\mathrm{NH}_{4}\right)_{2} \mathrm{HPO}_{4}$ apresentou melhor desempenho que o baseado em $\mathrm{H}_{3} \mathrm{PO}_{4}$. Isso sugere que a natureza do precursor de $\mathrm{P}$ pode alterar a natureza do sítio ativo do catalisador, de forma a modificar sua atividade na OPM. Em relação ao método de incorporação do $\mathrm{Fe}$, verificou-se que o catalisador sintetizado por troca iônica via radiação de micro-ondas $(0.5 \mathrm{FeHZB})$ foi menos ativo e estável que o sintetizado por impregnação úmida (0.5FeHZA). Entretanto, o catalisador $0.5 \mathrm{FeHZB}$ propiciou maiores produtividades instantâneas de formaldeído em alguns instantes da reação.

\section{AGRADECIMENTOS}

À Petrobras pelo fornecimento dos lotes de HZSM-5, ao NUCAT (PEQ/COPPE/UFRJ) pelas análises dos catalisadores e ao CNPq pelo apoio financeiro.

\section{REFERÊNCIAS}

BARBERO, J.A.; BAÑARES, M.A.; PEÑA, M.A.; FIERRO, J.L.G. Partial oxidation of methane into C1-oxygenates: role of homogeneous reactions and catalyst surface area. Catal. Today, v. 71, p. 1119, 2001.

BERRIER, E.; OVSITSER, O.; KONDRATENKO, E.V.; SCHWIDDER, M.; GRÜNERT, W.; BRÜCKER, A. Temperature-dependent $\mathrm{N}_{2} \mathrm{O}$ decomposition over Fe-ZSM-5: identification of sites with different activity. J. Catal., v. 249, p. 67-78, 2007.

BLASCO, T.; CORMA, A.; MARTÍNEZ-TRIGUERO, J. Hydrothermal stabilization of ZSM-5 catalytic-cracking additives by phosphorus addition. J. Catal., v. 237, p. 267-277, 2006.

FAJARDO, C.A.; NIZNANSKY, D.; N'GUYEN, Y.; COURSON C.; ROGER, A-C. Methane selective oxidation to formaldehyde with Fe-catalysts supported on silica or incorporated into the support. Catal. Commun., v. 9, p. 864-869, 2008.

FIERRO, G.; MORETTI, G.; FERRARIS, G.; ANDREOZZI, G.B. A Mössbauer and structural investigation of Fe-ZSM-5 catalysts: influence of $\mathrm{Fe}$ oxide nanoparticles size on the catalytic behaviour for the NO-SCR by $\mathrm{C}_{3} \mathrm{H}_{8}$. Appl. Catal. B, v. 112, p. 215-223, 2011.

KUMAR, M.S.; SCHWIDDER, M.; GRÜNERT, W.; BRÜCKENER, A. On the nature of different iron sites and their catalytic role in Fe-ZSM- 5 DeNO$_{x}$ catalysts: new insights by a combined EPR and UV/VIS spectroscopic approach. J. Catal., v. 227, p. 384-397, 2004.

LI, X.; SHEN, B.; XU, C. Interaction of titanium and iron oxide with ZSM-5 to tune the catalytic cracking of hydrocarbons. Appl. Catal. A, v. 375, p. 222-229, 2010.

WANG, Y.; WANG, X.; SU, Z.; GUO, Q.; TANG, Q.; ZHANG, Q.; WAN, H. SBA-15-supported iron phosphate catalyst for partial oxidation of methane to formaldehyde. Catal. Today, v. 93-95, p. 155-161, 2004. 